\title{
Use beam steering dipoles to minimize aberrations associated with off- centered transit through the induction bunching module. Design an improved NDCX-I drift compression section to make best use of the new bunching module to optimize planned initial NDCX-I target experiments
}

P. Seid11, J. Barnard2, F. Bieniosek1, J. Coleman1,3, D. Grote2, M. Leitner1, E. Gilson4, B.G.

Logan1, S. Lund2, S. Lidia1, P. Ni1, D. Ogata1,3, P. Roy1, W. Waldron1, D. Welch5, C. Wooton 1,3

\author{
1 \\ Lawrence Berkeley National Laboratory \\ 2 \\ Lawrence Livermore National Laboratory \\ 3 \\ University of California, Berkeley \\ 4 \\ Princeton Plasma Physics Laboratory \\ 5 \\ Voss Scientific \\ Accelerator Fusion Research Division \\ Ernest Orlando Lawrence Berkeley National Laboratory \\ University of California \\ Berkeley, California 94720
}

March 2008

This work was supported by the Director, Office of Science, Office of Fusion Energy Sciences, of the U.S.

Department of Energy under Contract No. DE-AC02-05CH11231. 
Report on research carried out by the Heavy Ion Fusion Science - Virtual National Laboratory in fulfillment of the FY08 Second Quarter Milestone, March 2008:

\title{
"Use beam steering dipoles to minimize aberrations associated with off-centered transit through the induction bunching module. Design an improved NDCX-I drift compression section to make best use of the new bunching module to optimize planned initial NDCX-I target experiments."
}

\author{
and the HIFS-VNL ${ }^{*}$ \\ ${ }^{1}$ Lawrence Berkeley National Laboratory \\ ${ }^{2}$ Lawrence Livermore National Laboratory \\ ${ }^{3}$ University of California, Berkeley \\ ${ }^{4}$ Princeton Plasma Physics Laboratory \\ ${ }^{5}$ Voss Scientific
}

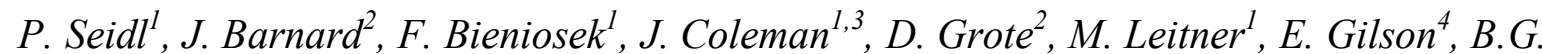

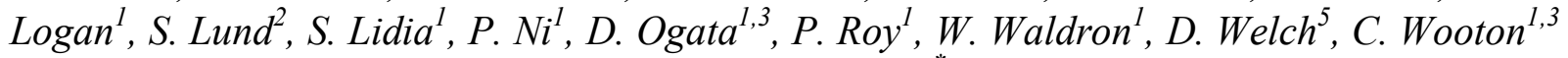

March 15, 2008

This milestone has been met by: (1) calculating steering solutions and implementing them in the experiment using the three pairs of crossed magnetic dipoles installed in between the matching solenoids, S1-S4. We have demonstrated the ability to center the beam position and angle to $<1 \mathrm{~mm}$ and $<1$ mrad upstream of the induction bunching module (IBM) gap, compared to uncorrected beam offsets of several millimeters and milli-radians. (2) Based on LSP and analytic study, the new IBM, which has twice the volt-seconds of our first IBM, should be accompanied by a longer drift compression section in order to achieve a predicted doubling of the energy deposition on future warm-dense matter targets. This will be accomplished by constructing a longer ferro-electric plasma source. (3) Because the bunched current is a function of the longitudinal phase space and emittance of the beam entering the IBM we have characterized the longitudinal phase space with a high-resolution energy analyzer.

\footnotetext{
* The US Heavy-Ion Fusion Science program has the long-term goal of developing inertialconfinement fusion as an affordable and environmentally attractive source of electrical power. Toward this goal, the near-term HIFS research at US National Laboratories uses reduced-scale experiments and state-of-the-art numerical simulations to understand the injection, transport, and focusing of the high-current beams needed for this approach to fusion energy. Since 1998, this research has been co-ordinated in the US by the Heavy-Ion Fusion Science Virtual National Laboratory, a collaboration between LBNL, LLNL and PPPL.
} 
Intense, space-charge-dominated ion beam pulses for warm dense matter and heavy ion fusion applications must undergo simultaneous transverse and longitudinal bunch compression in order to meet the requisite beam intensities desired at the target. NDCX has measured up to a 100-fold longitudinal current compression of an intense ion beam with pulse duration of a few nanoseconds [Coleman-PAC07].

\section{Beam steering results}

Misalignments of the solenoids in the transport lattice produce, to leading order, dipole magnetic field components that displace the transverse beam centroid from the design axis. Centroid oscillations resulting from these misalignments will tend to accumulate in a random walk sense as a function of the number of solenoids that the beam traverses. The total centroid excursion includes contributions from these misalignment effects and any initial errors of the beam being injected off-axis into the transport lattice. These effects may also be exacerbated by energy variations along the beam pulse. Measurements at the end of the solenoid transport lattice of the NDCX indicate typical centroid excursions with an amplitude of a few $\mathrm{mm}$ after going through the four solenoids of the transport lattice.

Such centroid excursions in the NDCX are large enough to cause problems in downstream final-focus applications and must be corrected. This has been modeled in LSP simulations by injecting otherwise ideal beams into the induction bunching module gap with offsets of a few $\mathrm{mm}$. The consequence on peak energy deposition on the target is $>35 \%$ for an offset of $2 \mathrm{~mm}$ [Welch-PAC07]. The simulations suggest that corrections of the beam offset to $<0.5 \mathrm{~mm}$ would be desireable. 
Specific values of the phase-space coordinates at the measurement plane depend on the machine operating point employed (set of solenoid strengths, and beam energy). A particular example of centroid errors measured is given in the "Dipoles off" row of Table 1.

\begin{tabular}{|l|c|c|c|c|}
\hline & $\begin{array}{c}\langle\mathbf{x}\rangle \\
{[\mathbf{m m}]}\end{array}$ & $\begin{array}{c}\left\langle\mathbf{x} \mathbf{\prime}^{\prime} \mathbf{c}\right. \\
{[\mathbf{m r a d}]}\end{array}$ & $\begin{array}{c}\langle\mathbf{y}\rangle \\
{[\mathbf{m m}]}\end{array}$ & $\begin{array}{c}\left\langle\mathbf{y}^{\prime}\right\rangle \\
{[\mathbf{m r a d}]}\end{array}$ \\
\hline Dipoles off & -5.81 & 2.24 & -2.77 & 3.37 \\
Dipoles on & -0.15 & 0.51 & 0.00 & -0.31 \\
\hline
\end{tabular}

Table 1: Results of steering dipole measurements. First moments of the beam position and angle are measured at the exit of the four-solenoid matching section, just before the induction bunching module.

To accomplish the centroid corrections, three cross-field magnetic steering dipoles were installed in the lattice between the solenoids as indicated in Fig. 1. Individual dipoles were designed to apply up to 20 milliradian kicks to the $300 \mathrm{keV} \mathrm{K}{ }^{+}$beam. The y-field dipoles (for x-plane bends) are are composed of 78 turns of 1-mm copper wire, in a uniform current density $\left(\mathrm{K}(\theta)=\right.$ constant for $\left.-60<\theta<60^{\circ}\right)$ approximation to a $\cos (\theta)$ coil. The coil radius and axial length are $51.3 \mathrm{~mm}$ and $52.1 \mathrm{~mm}$. A similar $\mathrm{x}$-field dipole (for $\mathrm{y}$-plane bends) with a slightly different radius $(47.5 \mathrm{~mm})$ and length $(71.1 \mathrm{~mm})$ coil are nested to provide angular kicks in the y-plane. A CAD model of the coils is shown in Fig. 2, and photographs of the assembled dipole set is shown in Fig. 3. Note that the full steering assembly fits in the 10-cm gaps between solenoids and around the $100 \mathrm{~mm}$ diameter beam pipe. More than the minimum of two steering dipoles per plane were placed in the lattice to allow for extra flexibility in tuning out errors as the beam evolves in the lattice. 
Report on research carried out by the HIFS-VNL in fulfillment of the FY08 Q2 Milestone

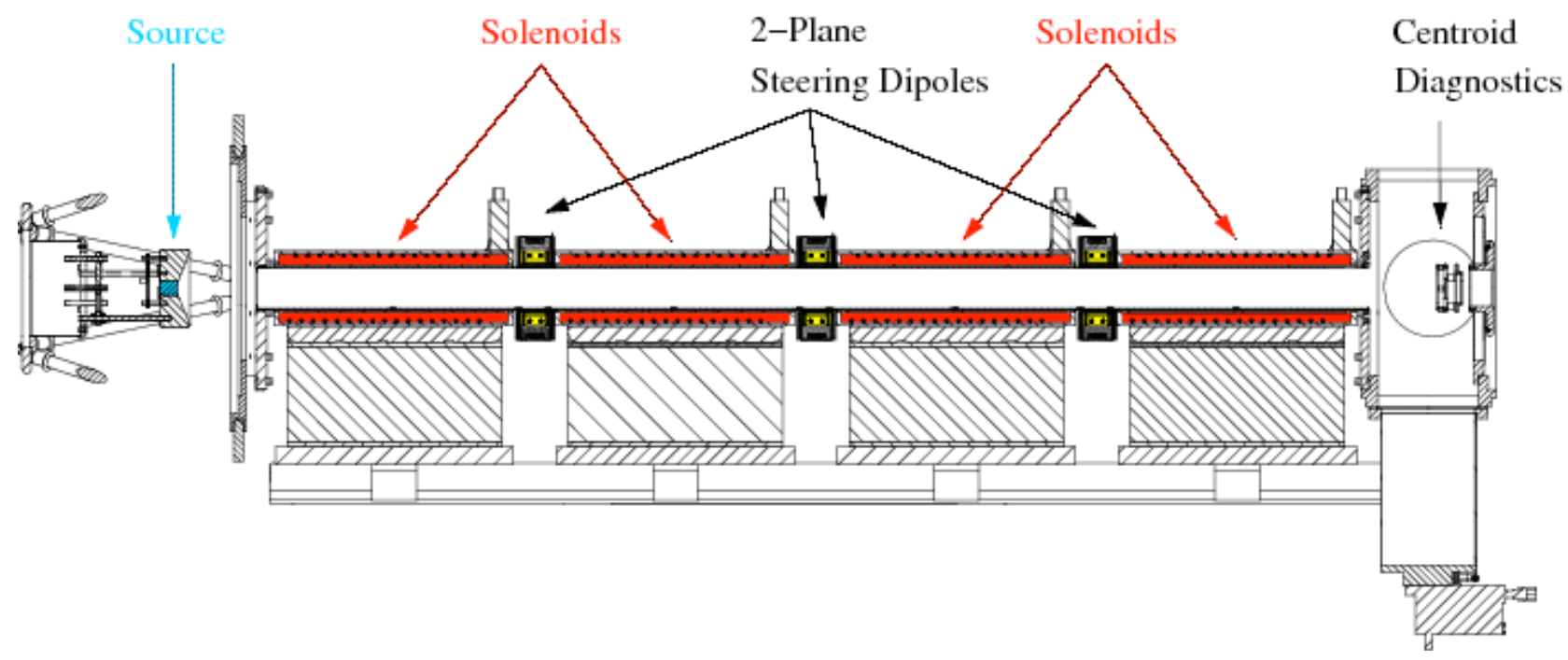

Figure 1: NDCX solenoid transport lattice with the location of solenoids and steering dipole assemblies indicated.

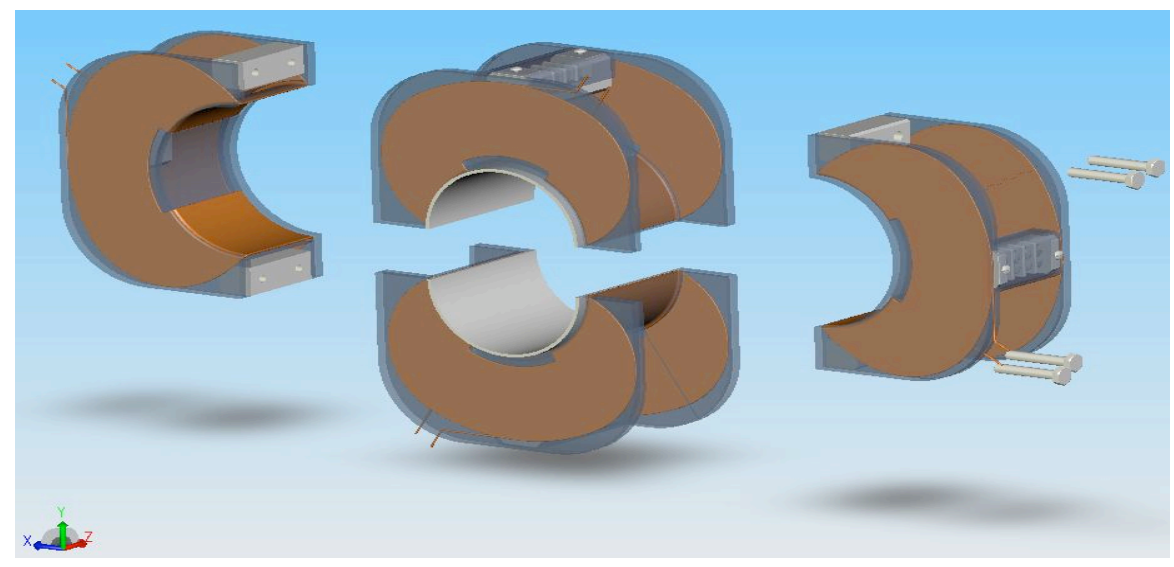

Figure 2: Exploded view of the CAD model of a steering dipole assembly shows how the horizontal and vertical coils are nested. 


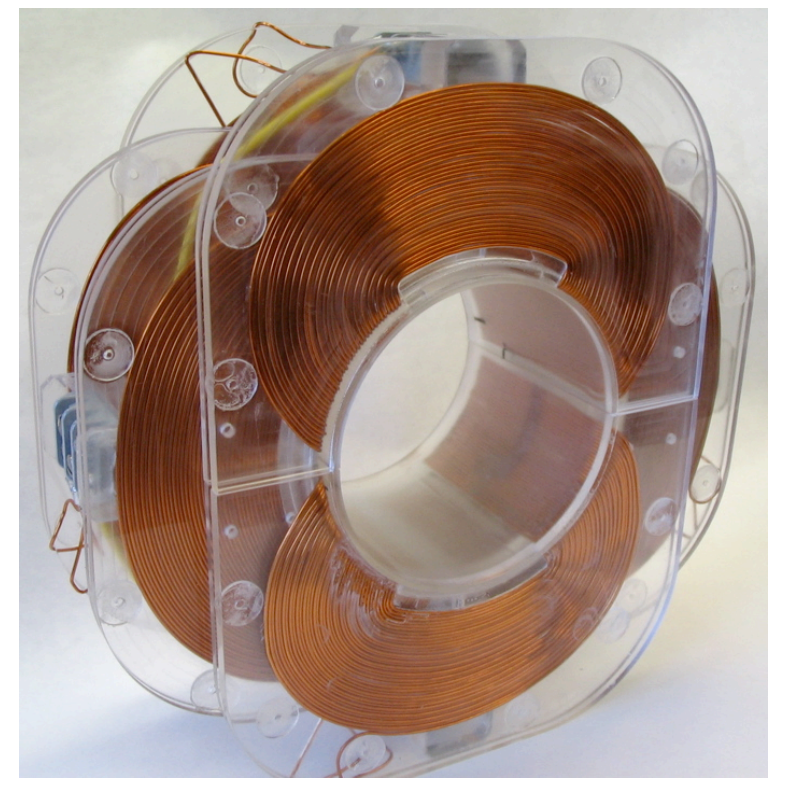

Figure 3: Photo of a horizontal and vertical steering dipole assembly.

The steering dipole coils are driven by pulse forming network pulsers, with a pulse duration of $2 \mathrm{msec}$. The field strength generated is approximately constant over the several $\mu$ s beam pulse. The peak voltage and current amplitude are $\sim 200$ Volts and $\sim 200$ Amps. The magnetic field produced is $\sim 4.0$ and $\sim 2.8$ Gauss/Amp for the $\mathrm{x}-$ field and y-field coils, respectively. This has been confirmed with approximate, analytical models. Thus, a current of $100 \mathrm{~A}$ creates a dipole field of $\sim 0.03$ Tesla and a deflection to the $0.3 \mathrm{MeV} \mathrm{K}{ }^{+}$ions of $\sim 6 \mathrm{mrad}$.

Steering corrections in solenoid transport lattices are complicated because the centroid orbit Larmor precesses due to the strong axial magnetic field, inducing an effective rotation on the centroid evolution. Rotational phase accumulations of $\sim$ 180 degrees are characteristic of NDCX experiments. Thus, dipole kicks acting at the corrector positions in $\mathrm{x}$-plane will be projected into both the $\mathrm{x}$-and $\mathrm{y}$-planes as the centroid evolves downstream of the correction kick. The dipoles sit in the solenoid fringe field regions, which add further complexity to the correction scheme. 
A practical, fully laboratory-based correction procedure has been derived and successfully carried out to deal with this complicated tuning issue to null the centroid errors. A low-current ("pencil") beam is employed near the matching section tunes of interest. Currents of each dipole corrector coil in the $2^{\text {nd }}$ and $3^{\text {rd }}$ steering modules are independently varied and the full centroid phase-space response of the centroid is measured for each current variation. This procedure effectively builds up a $4 x 4$ Jacobian matrix of the linear system response to dipole current excitations. This matrix is then applied to numerically calculate currents (ranging from 56 to 194 Amps) necessary to null the centroid error. Application of these currents to the dipoles produced the corrected centroid shown in the "Dipoles on" column of Table 1. Note that the correction appears perfect to the measurement uncertainty of 0.25 $\mathrm{mm}$ and $1 \mathrm{mrad}$ in the centroid coordinates and angles. This good result essentially verifies that nonlinear effects are negligible relative to measurement uncertainties.

The virtue of the Jacobian-based experimental correction procedure is that is is formulated directly in terms of laboratory variables and measurements. Thus, it automatically takes into account sign conventions and procedures employed with no ambiguity. The correction coming out to measurement accuracy verifies that beam control is fully achievable and enabled us to meet performance requirements.

Unfortunately, the Jacobian-based procedure is also laborious. It requires full phase space measurements to be accumulated for each of the four independent current variations. This tuning would also have to be repeated every time the solenoid field strengths upstream are adjusted significantly since the system response to the alignment errors will change. Also, the data for Jacobian responses must avoid beam scraping when carried out with the full beam. 
Due to these limitations, a more theory-based procedure is being developed that promises to vastly reduce the labor involved in correcting the beam centroid: An ideal solenoid field model is displaced and tilted (pitch and yaw angles) about it's centerline. The resulting field is resolved into an ideal component plus dipole terms related to the misalignment parameters. Equations of motion are then derived for the transverse centroid evolution. These equations of motion are expressed in the rotating Larmor frame where they are most simply expressed. An analogy to dispersion functions is then exploited to derive a linear, small-amplitude expansion of the centroid orbit in terms of 3 components:

1) The oscillation due to initial condition errors subsequently evolving in the ideal lattice without alignment errors or steering.

2) The oscillation due to mechanical misalignments of the solenoid. This is expressed in terms of displacement and magnet tilt functions for each solenoid that are properties of the ideal (aligned) lattice alone times the corresponding misalignment amplitudes.

3) The oscillation due to dipole steering. This is expressed in terms of steering functions for each dipole that are properties of the ideal (aligned) lattice alone times the corresponding bending dipole strength.

The structure of this centroid orbit expansion can be exploited to systematize steering corrections based on fewer (rather than four for the Jacobian method) phasespace measurements. Also, for sufficiently accurate experimental data, it can be exploited with minimally or over-constrained data sets to calculate the actual misalignment parameters of the lattice both in terms of the initial condition error of the beam emerging from the injector ( 4 parameters) and the mechanical and the 
transverse rotational misalignments of each solenoid ( 4 parameters per solenoid). Such detailed information on the actual misalignments of the lattice is novel within accelerator physics and, in principle, enables subsequent mechanical correction of the lattice and/or incorporation of the misalignment parameters in detailed simulations for improved system modeling.

At present, procedures to exploit this expansion for more efficient steering corrections are underway in the laboratory. Preliminary indications are encouraging, but it is not yet clear whether the final corrections will be effective to sufficient accuracy.

Also, the formulation has been applied to better understand the statistical contributions of various errors to characteristic centroid oscillations. In Fig. 4, the rms $x$-centroid $(x=<x>)$ is shown due to an ensemble of 10,000 random error sets uniformly distributed up to cut-off values. The errors in the initial coordinate are cut-off at $2 \mathrm{~mm}$ and $5 \mathrm{mrad}$, and solenoid alignment errors are cutoff at $1.5 \mathrm{~mm}$ and $5 \mathrm{mrad}$ in transverse displacement and tilt angle, respectively. Curves indicate rms contributions (add in quadrature) due to all errors (red), solenoid alignment displacement + tilt alignment (magenta), tilts only (blue), displacements only (green), and initial condition errors (black). These characteristic expected values of misalignment parameters produce reasonable agreement in magnitude with measured centroid errors at a similar operating point (see the "Dipoles off" row of Table 1). This study has indicated that the mechanical tilt alignment of the solenoids is more important than previously anticipated. 
Report on research carried out by the HIFS-VNL in fulfillment of the FY08 Q2 Milestone
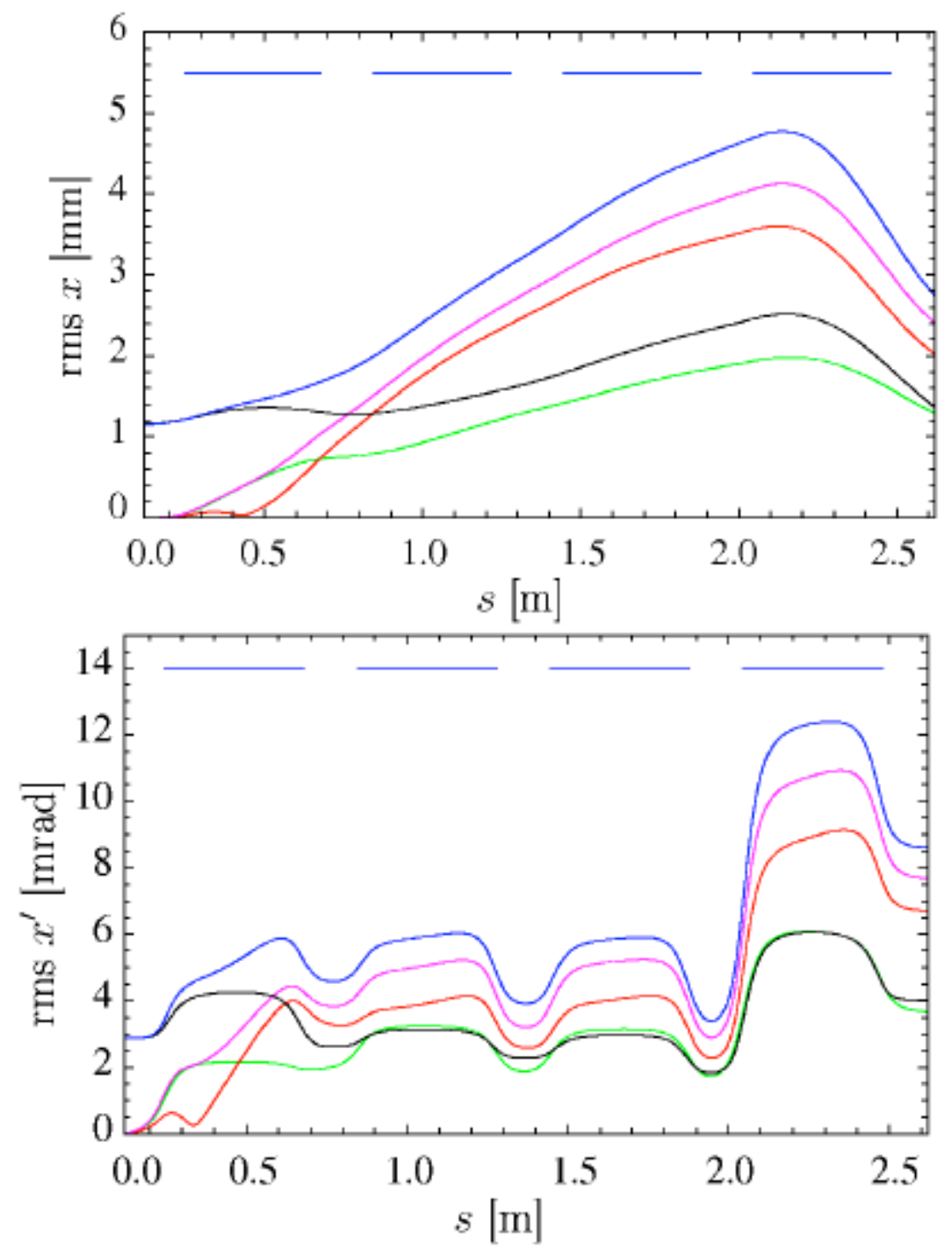

Figure 4: Statistical contribution of error sources to rms centroid misalignments for $\mathrm{x}$-position $(\mathrm{x}=<\mathrm{x}>)$ above and $\mathrm{x}$-angle $\left(\mathrm{x}^{\prime}=<\mathrm{x}^{\prime}>\right)$ below.

\section{Beamline design for the new induction bunching module}

The new bunching module is shown in Fig. 5. It has 20 induction core units, (vs the presently utilized 12 core units), or nearly twice the volt-seconds available compared to the present setup. The experiment design question is whether the 
increase capability should be used, for example, to impart a higher velocity tilt, $\Delta=$ $\delta v / v_{0}$, (higher peak voltage), or keeping it unchanged for a shallower slope of the tilt and a correspondingly longer drift compression length.

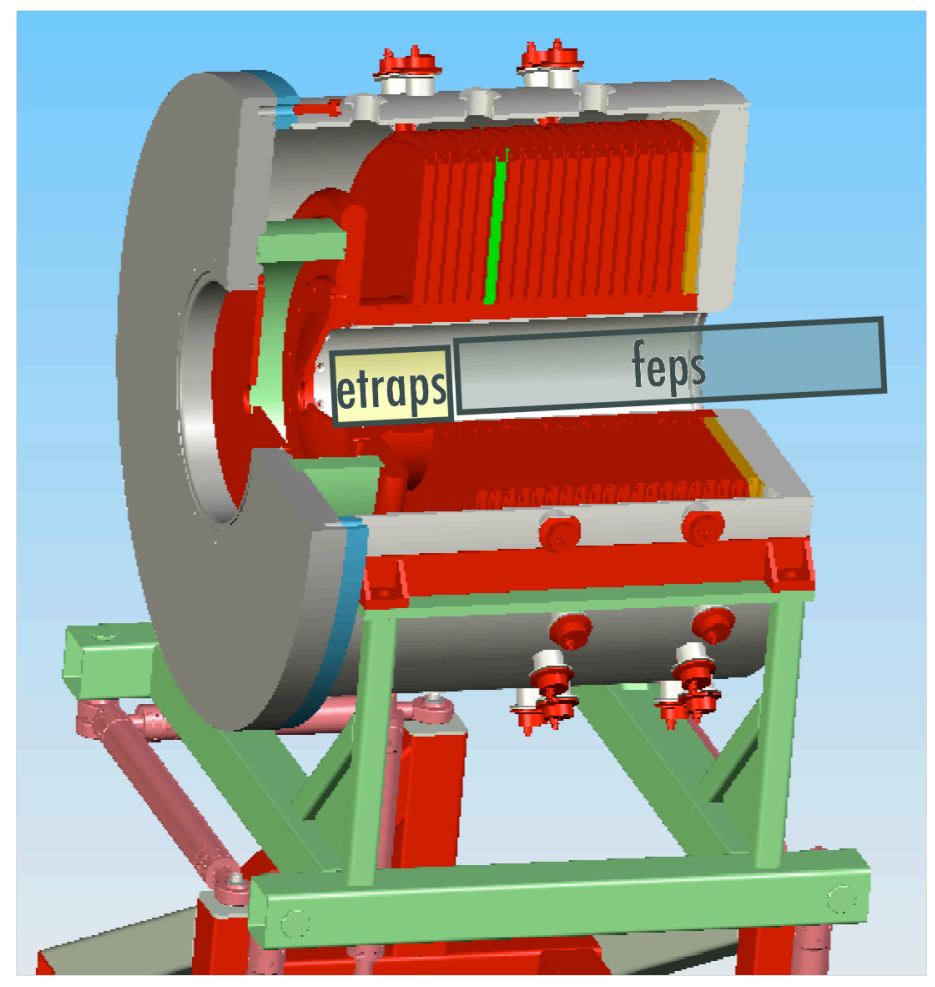

Figure 5: The new induction bunching module has 20 cores which will be individually tuned and driven by pulsers to achieve an overall waveform to bunch the beam at the target diagnostics plane.

The trade-offs are between a shorter focused pulse duration (with greater chromatic aberrations), or a longer pulse duration with fewer chromatic aberrations. The energy deposition on the target is approximately [Barnard-07]:

$$
\mathrm{E}=\frac{4 e \phi I \tau}{\pi \varepsilon f \Delta} F_{1}(\eta) \tan ^{-1}\left(\frac{F_{2}(\eta) r_{0}^{2} \Delta}{2 f \varepsilon}\right) \underset{\sim}{\propto} \frac{e \phi I \tau}{\varepsilon f \Delta}
$$


where $e \phi$ is the ion kinetic energy, $I$ is the beam current before compression, $\tau$ is the initial pulse duration before compression, $\varepsilon$ is the un-normalized transverse emittance of the beam, $f$ is the focal length of the final focusing solenoid, $r_{0}$ is the radius of the beam on entrance to the solenoid, and $F_{1}(\eta)$ and $F_{2}(\eta)$ are functions that depend only on the ratio $\eta$ of the length of the solenoid to the focal length of the solenoid. (For NDCX1, $\eta=.43$, and $F_{l}(.43)=1.01$ and $F_{2}(.43)=1.24$ ). Equation (1) is based on assuming that all slices of the beam enter the solenoid at radius $r_{0}$ independent of velocity. Chromatic aberrations' influence on the spot radius is proportional to $(\Delta)^{1 / 2}$. Thus, keeping the focal length approximately the same in the experiment (attractive because of the simplicity), and increasing $\Delta$ and $\tau$ to use the additional volt seconds appear to cancel from the above scaling. Conversely, doubling the $\tau$, keeping $\Delta$ fixed, and doubling the drift compression distance (distance from the IBM gap to the target plane) approximately doubles E, and doubles the compressed pulse duration under the assumption of conservation of longitudinal emittance.

We tested these scaling arguments with a series of analytic approximations and LSP simulations which include several additional experimental constraints:

1) The range of initial beam envelope conditions at the entrance to the IBM is constrained by the beam pipe diameter and solenoid field strengths in the matching section.

2) In addition to the desired velocity ramp, the IBM imparts a time dependent defocusing in the transverse plane, dispersing the beam envelope at the entrance to the final focusing solenoid, compounding the chromatic aberrations. 
3) The initial conditions are also constrained by the beam pipe radius in the final focusing solenoid.

Note that we are studying methods of correcting the time-dependent defocusing and chromatic effects of the IBM. A pulsed electric Einzel lens or quadrupole doublet can likely meet the requirements. We will report on the comparison of theoretical models and initial measurements of focal spot reductions using upstream time-dependent corrections of chromatic aberrations in FY09.

The analytic model is based on the formulae for the IBM waveform for a finite length, fully neutralized beam drift compressing to a longitudinal emittance-limited bunch. Transverse envelopes were calculated for multiple energy slices, using the $\mathrm{KV}$ envelope equation. The beam distributions for each energy slice, assumed to be Gaussian near the focal plane, were summed to estimate the peak E. An example of the transverse envelopes for multiple energy slices is shown in Fig. 6.

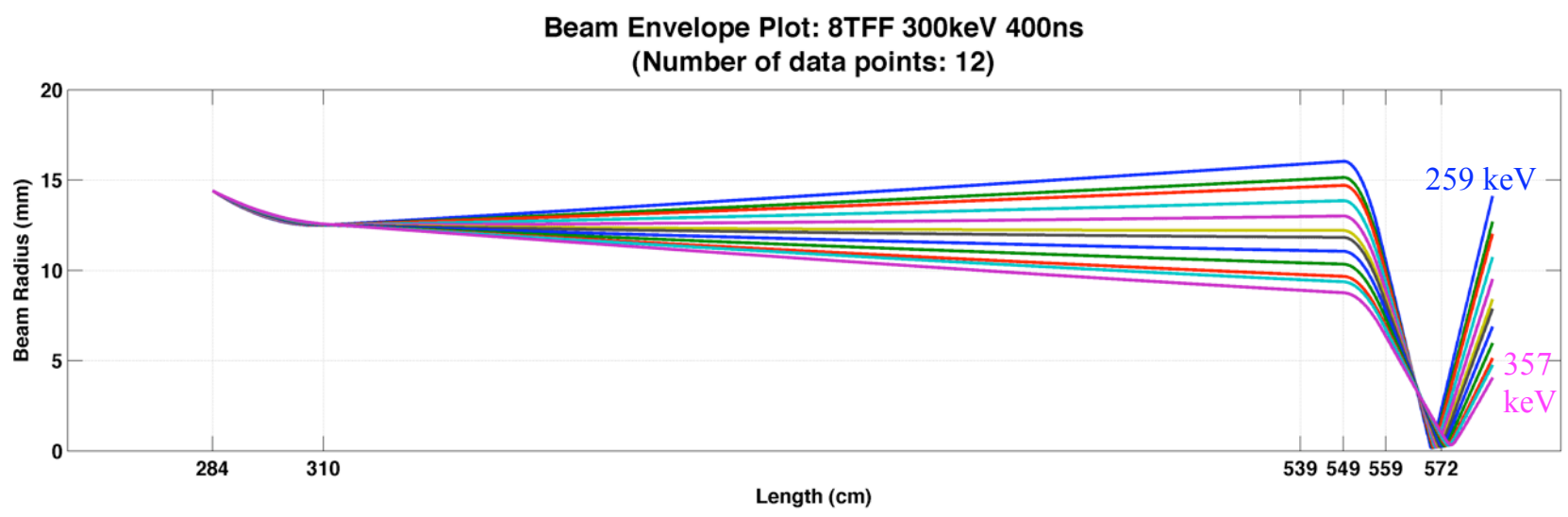

Figure 6: The calculation is initialized at $\mathrm{z}=284 \mathrm{~cm}$, at the exit of the IBM gap. The envelope slices are calculated assuming full space charge contribution, until the entrance to the FEPS at $\mathrm{z}=310 \mathrm{~cm}$. The beam propagates assuming full neutralization through the final focusing solenoid $(549<\mathrm{z}<559 \mathrm{~cm})$ to the focal plane $(\mathrm{z}=572 \mathrm{~cm})$. 
The sweeping of the focal plane about the desired location at $\mathrm{z}=572 \mathrm{~cm}$ due to the energy dispersion is evident in Fig. 6.

The time-dependent velocity $\operatorname{ramp}, v(t)$, that compresses the beam at a downstream distance $\mathrm{L}$ with a leading edge velocity $v(0)$, is:

$$
v(t)=\frac{v(0)}{(1-v(0) t / L)}
$$

and the IBM voltage waveform producing this ramp, applied in the envelope slice model as well as in the LSP simulations, is:

$$
V(t)=\frac{1}{2} m v^{2}(t)-\phi_{o}
$$

$e \phi_{o}$ is the ion kinetic energy. Several calculations were carried out where $\tau$ and initial beam envelope were varied parametrically in LSP and in the slice-envelope calculations. A few are highlighted in Table 1, which illustrate our observations and

\begin{tabular}{|c|c|c|c|c|c|c|c|c|c|c|}
\hline & $\begin{array}{l}\mathbf{F F} \\
(\mathbf{T})\end{array}$ & $t$ (ns) & $\begin{array}{c}\text { initial } \\
\text { kinetic } \\
\text { energy } \\
(\mathrm{keV})\end{array}$ & $\begin{array}{c}a(z=284) \\
(\mathrm{mm})\end{array}$ & $\begin{array}{c}\mathbf{a}^{\prime} \\
(\mathrm{mrad})\end{array}$ & $\begin{array}{l}\text { Current } \\
\text { at focus } \\
\text { (Amps) }\end{array}$ & $\begin{array}{c}\text { pulse } \\
\text { width @ } \\
\text { focus } \\
\text { (ns) }\end{array}$ & $\begin{array}{l}E(\mathrm{~J} / \mathrm{cm} 2) \\
\text { envelope }\end{array}$ & $\begin{array}{c}E(\mathrm{~J} / \mathrm{cm} 2) \\
\text { LSP2 }\end{array}$ & $\begin{array}{c}E(J / c m 2) \\
(E q .1)\end{array}$ \\
\hline a) & 0 & 200 & 300 & 21.50 & -23.80 & 3.08 & 1.69 & 0.06 & & \\
\hline b) & 8 & 282 & 300 & 9.55 & -9.82 & 4.01 & 1.83 & 0.39 & 0.30 & 0.59 \\
\hline c) & 8 & 400 & 300 & 14.40 & -13.70 & 3.23 & 3.22 & 0.82 & 0.69 & 0.94 \\
\hline
\end{tabular}
conclusions.

Table 2: Comparison of LSP, the envelope-slice model, and the simple analytic scaling relation (Eq. 1). The first row (a) corresponds to experiments without a final focusing solenoid. The second and third rows corresponds beamlines using the new 
IBM, the final focusing solenoid $\left(\mathrm{B}_{\max }=8\right.$ Tesla $)$ and two beamline configurations: (b) with the present drift compression length $(\mathrm{L}=144 \mathrm{~cm})$, and (c) with twice the drift compression length $(\mathrm{L}=288 \mathrm{~cm})$ as the present setup.

We have noted that the overall magnitude of the energy deposition calculations is overestimated by the model calculations compared to NDCX data without a final focusing solenoid. For example, in case (a), the 2D LSP results are a factor of 3-4 greater than energy deposition observed in the lab. This discrepancy is not yet resolved, but LSP simulations suggest that parametric variations of plasma density, and beam centroid offsets (which enhance the sampling of aberrations in the IBM fields) may be responsible.

It is clear that case (c) in gives the highest energy deposition $(0.69 \mathrm{~J} / \mathrm{cm} 2$ in the LSP simulations, $>2 \mathrm{x}$ greater than (b). This is at the expense of a longer pulse duration (3.2 ns FWHM), but satisfactory for initial warm dense matter target experiments. The LSP results for case (c) are shown in Fig. 7.
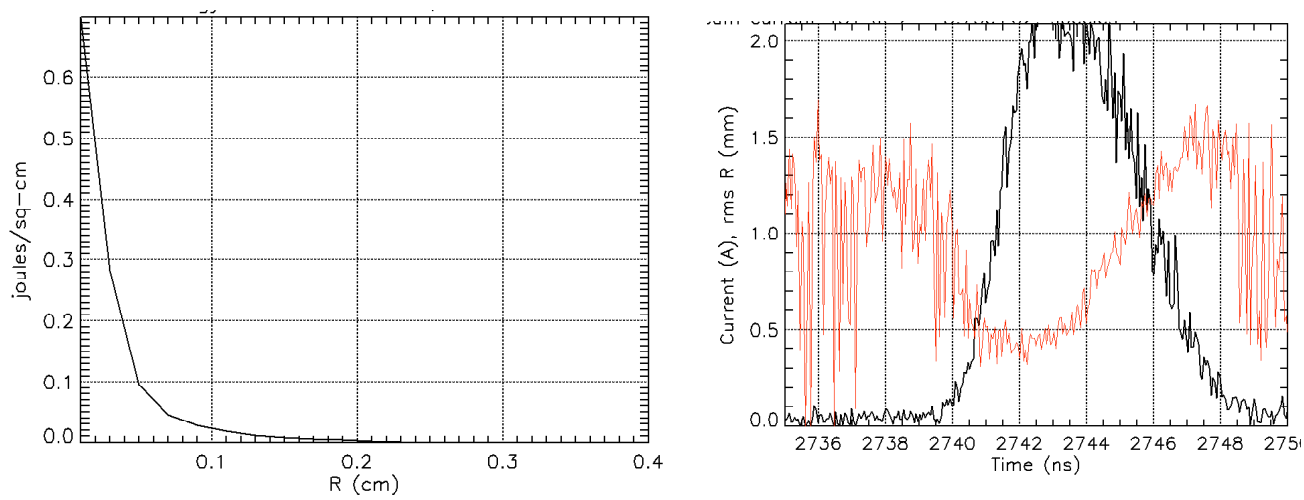

Figure 7: LSP simulations predict a peak energy deposition of $0.69 \mathrm{~J} / \mathrm{cm}^{2}$ at the focal plane (left). In the right plot, the predicted peak current (black) is $2.1 \mathrm{~A}$, with a minimum radius of $0.4 \mathrm{~mm}$ (red). 
Thus, we have concluded that it is advantageous to lengthen the drift compression section by $1.44 \mathrm{~m}$ via extension of the FEPS. A drawing showing the planned modification of the beamline is shown in Fig. 8. The present FEPS is $85 \mathrm{~cm}$ long. All of the barium titanate rings and most of the ancillary components are in hand.

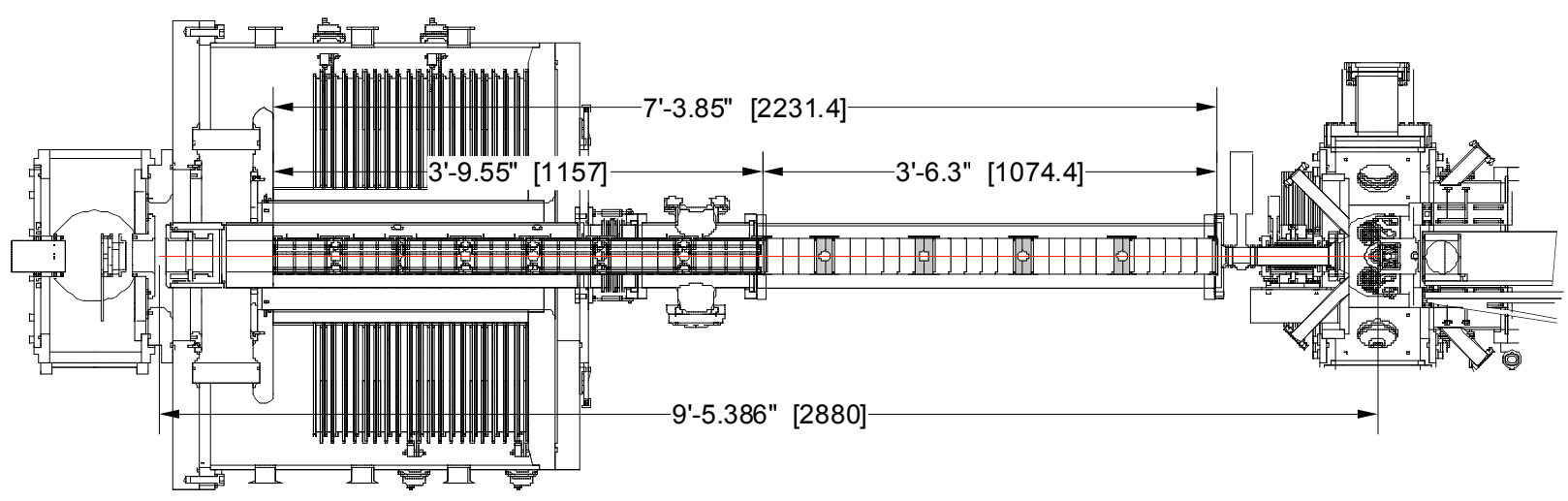

Figure 8: Drawing showing the NDCX beamline modifications to make best use of the new IBM. Units in [] are millimeters.

\section{Measurement of the energy spread of the NDCX beam before bunching}

In order to constrain bunch compression modeling, we have recently used the new high-resolution electrostatic energy analyzer to study the energy spread of the beam in the absence of the IBM velocity ramp. For example, previous simulations have used the longitudinal emittance as an adjustable parameter, and found that $\mathrm{T}_{\|} \sim 0.2$ $\mathrm{eV}$ gives fair agreement with the NDCX measurements by comparing the compressed pulse bunch duration to the experimental results. We report here a preliminary analysis of the measured energy spread of the injected beam with the energy analyzer (Fig. 9). 


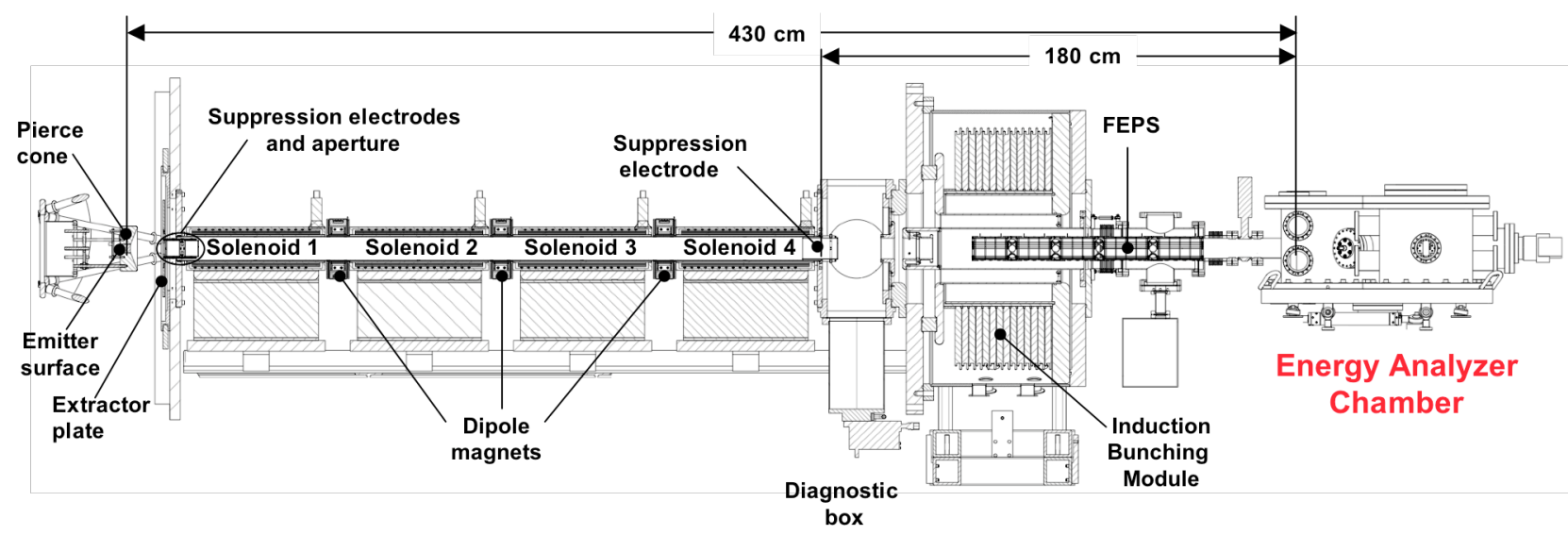

Figure 9: Beamline setup for the electrostatic energy analyzer experiments. The IBM was not energized for these measurements. The energy analyzer included a Faraday slit collector, an alumina scintillator and II-CCD or a streak camera for time dependent measurements of the scintillator on single shots.

A detailed calibration of the DC power supply voltages to the deflecting plates preceded the beam measurements, along with alignment checks with a laser tracking alignment system. For the beam measurements, a $0.1-\mathrm{mm}$ × $5 \mathrm{~mm}$ slit aperture defined the incoming beam to the electrostatic spectrometer. Its width defines a minimum energy resolution of the spectrometer to be $\Delta \mathrm{E}=60 \mathrm{eV}$ (FWHM). Space charge and field aberrations in the spectrometer were estimated to be insignificant for most of the measurements. The acceptance of the spectrometer is $\approx 10 \mathrm{keV}$ for an incoming $\mathrm{K}^{+}$kinetic energy (central trajectory) of $300 \mathrm{keV}$. Measurements were made with three diagnostic systems: (i) a scintillator at the focal plane of the spectrometer was imaged with an image-intensified gated CCD camera for gate widths of 50-500 ns at various locations through the 3- $\mu$ s long beam pulse; (ii) the same scintillator, imaged with a streak camera, apertured to an effective time resolution of $0.25 \mu \mathrm{s}$; (iii) a slit Faraday collector at the focal plane measured the transmitted charge. The energy distribution was measured by repeating beam shots and varying the deflecting plate voltages. 
The measured energy spread, $\Delta \mathrm{E}$ over a finite time interval, has contributions from systematic (short and long) time variations of the Marx voltage waveform, instrumental resolution and the random (or thermal) energy spread of the beam. In the limit that thermal effect dominates or is unfolded from the data, then $\Delta E_{t h}$ is related to the beam longitudinal temperature by [Reiser-1994]:

$$
\Delta E_{t h}=\sqrt{2 E T_{\|}}
$$

The experimental results are summarized in Fig. 10.
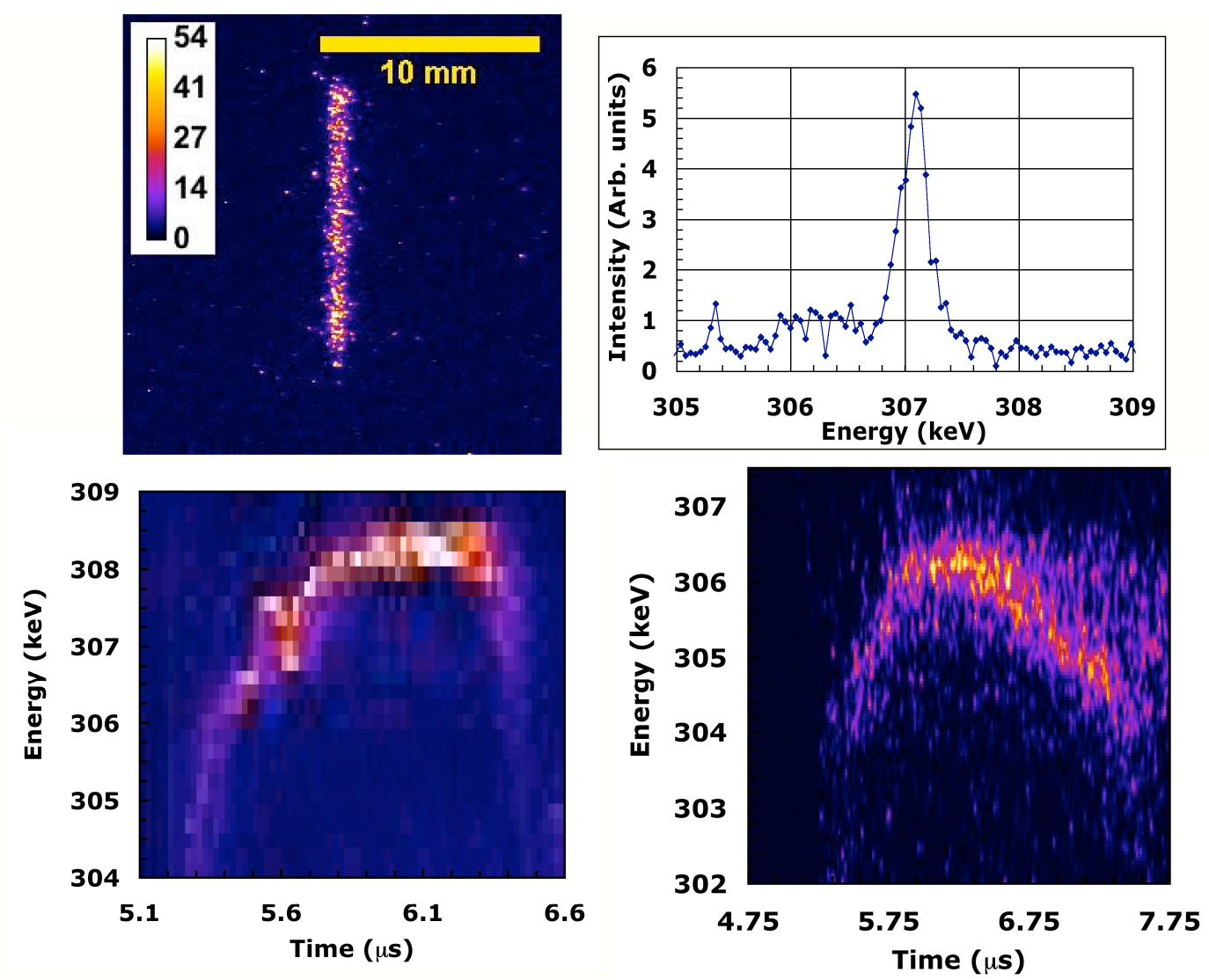

Figure 10: Clockwise from top left: Example of a beam image at focal plane of the energy analyzer, collected by the II-CCD camera. Energy distribution at the focal plane as measured on a single pulse with the same setup, with a $0.1-\mu$ s gate 
near the flat-top of the beam energy distribution. Longitudinal phase space measured with the streak camera (average of 10 pulses). Longitudinal phase space measured with the slit Faraday collector (composite of numerous beam pulses).

The slit Faraday collector measurements have much better time resolution, but the observed phase space energy spread is broadened by shot-to-shot energy variations $(\approx 1 \mathrm{keV} \mathrm{rms})$ due to Marx voltage waveform fluctuations.

Based on an average of 40 individual beam pulse images, the energy spread at the focal plane is $\Delta \mathrm{E}=0.113 \mathrm{keV} \pm 0.037 \mathrm{keV}$ (corresponding to $\mathrm{T}_{\|}=0.023 \mathrm{eV}$ ). We note that they are indeed lower than the longitudinal emittance empirically adjusted to match the NDCX simulations to date but more in-line with integrated LSP simulations which predicted $\mathrm{T}_{\|}=0.01 \mathrm{eV}$ [Welch-PAC07]. Due to these ongoing investigations of instrumental effects, we are treating the observed energy spread and effective beam temperatures as an upper limit. This more precise measurement of the longitudinal phase space constrains the models, and allows us to separate effects due to the initial emittance from effects coming from IBM waveform imperfections and beam plasma interactions.

Warp PIC modeling of these energy analyzer experiments are underway. We are studying systematic contributions to the observed $\Delta \mathrm{E}$, such as (i) field aberrations in the energy analyzer due to finite misalignments of the components, (ii) space charge effects, and (iii) Marx waveform variations which are averaged over the gate times (0.1-0.5 $\mu \mathrm{s})$.

\section{References:}

[Welch-PAC07] Proceedings of PAC07, Albuquerque, NewMexico, USA, D.R. Welch et al., End-To-End Simulations Of An Accelerator For Heavy Ion Beam Bunching, http://accelconf.web.cern.ch/AccelConf/p07/PAPERS/THPAN086.PDF 
[Coleman-PAC07] J.E. Coleman et al., Bunching And Focusing Of An Intense Ion Beam For Target Heating Experiments, http://accelconf.web.cern.ch/AccelConf/p07/PAPERS/THPAS004.PDF

[Barnard-07] "Update on requirements for Target Experiments on NDCX II', http://hifweb.lbl.gov/public/slides/Barnard.NDCX.2007.11.07.pdf

(also, J.J. Barnard et al, "Accelerator and Ion Beam Trade Offs for Study of Warm Dense Matter," Proceedings of Particle Accelerator Conference, RPAP039 (2005).

[Reiser-94], "Theory and Design of Charged-Particle Beams," [Wiley, NJ], (1994). 